\title{
32. A Theorem for Metrizability of a Topological Space
}

\author{
By Jun-iti Nagata \\ Department of Mathematics, Osaka City University \\ (Comm. by K. Kunugi, M.J.A., March 12, 1957)
}

Since Alexandroff and Urysohn's work various theorems concerning metrizability of a topological space were gotten by many mathematicians, but their methods of proofs are, in general, various and rather complicated. The purpose of this brief note is to prove a theorem for metrizability, which will contain a large number of metrizability theorems as direct consequences. ${ }^{1)}$

We use the following theorem due to $\mathrm{E}$. $\mathrm{Michael}^{2)}$ as well as the well-known theorem of P. Alexandroff and P. Urysohn.

Michael's theorem. A regular topological space $R$ is paracompact if and only if every open covering of $R$ has an open refinement $\mathfrak{B}=\stackrel{\infty}{n=1}_{\mathfrak{B}}$, where each $\mathfrak{B}_{n}$ is a locally finite collection of open subsets of $R$.

Theorem 1. In order that a $T_{1}$-topological space $R$ is metrizable it is necessary and sufficient that one can assign a nbd (=neighborhood) basis $\left\{U_{n}(x) \mid n=1,2, \cdots\right\}$ for every point $x$ of $R$ such that for every $n$ and each point $x$ of $R$ there exist $n b d s S_{n}^{1}(x), S_{n}^{2}(x)$ of $x$ satisfying

i) $y \notin U_{n}(x)$ implies $S_{n}^{2}(y) \frown S_{n}^{1}(x)=\phi$,

ii) $y \in S_{n}^{1}(x)$ implies $S_{n}^{2}(y) \subseteq U_{n}(x)$.

Proof. Since the necessity is clear, we prove only the sufficiency. To begin with, $R$ is regular, since $\bar{S}_{n}^{1}(x) \subseteq U_{n}(x)$. Next, to show that $R$ is paracompact, we take an arbitrary open covering $\mathfrak{B}=\left\{V_{\alpha} \mid \alpha<\tau\right\}$ of $R^{3)}$ If we let

$$
\begin{gathered}
\left.V_{n \alpha}=\smile\left\{\left(S_{n}^{1}(x)\right)^{\circ} \mid U_{n}(x) \subseteq V_{\alpha}\right\},{ }^{4}\right) \\
V_{m n \alpha}=\smile\left\{U_{m}(x) \mid U_{m}(x) \subseteq V_{n \alpha}\right\}, \\
V_{m n \alpha}^{\prime}=\smile\left\{S_{m}^{1}(x) \mid U_{m}(x) \subseteq V_{n \alpha}\right\}, \\
M_{m n \alpha}=\left(V_{m n \alpha}^{\prime}-\smile V_{\beta<\alpha}\right)^{\circ} \quad(m, n=1,2, \cdots, \alpha<\tau),
\end{gathered}
$$

then $\mathfrak{M}_{m n}=\left\{M_{m n \alpha} \mid \alpha<\tau\right\}$ is a locally finite open collection for each $m, n$. To show the local finiteness of $\mathfrak{M}_{m n}$ we choose, for an arbitrary point $p$ of $R, \alpha(\leqq \tau)$ such that $p \in V_{m n \alpha}, p \notin V_{m n \beta}(\beta<\alpha)$. Then it follows from the condition i) of the proposition that $S_{m}^{2}(p) \frown V_{m n \beta}^{\prime}=\phi$

1) The detail of the content of this note will be published in an another place.

2) See [3].

3) In this proof we denote by $\tau, \alpha, \beta, \gamma$ ordinal numbers.

4) $A^{\circ}$ denotes the interior of $A$. 
$(\beta<\alpha)$, which implies $S_{m}^{2}(p) \frown M_{m n \beta}=\phi \quad(\beta<\alpha)$. Since $p \in V_{m n \alpha} \subseteq V_{n \alpha}$ and $V_{n \alpha}$ is open, we obtain a nbd $V_{n \alpha}$ of $p$ satisfying $V_{n \alpha} \frown M_{m n r}=\phi$ $(\gamma>\alpha)$. Therefore the nbd $S_{m}^{2}(p) \frown V_{n \alpha}$ of $p$ intersects at most one of elements of $\mathfrak{M}_{m n}$, proving the local finiteness of $\mathfrak{M}_{m n}$.

To assert that $\underset{m, n=1}{\infty} \mathfrak{M}_{m n}=\mathfrak{M}$ covers $R$, we consider an arbitrary point $p$ of $R$. Let $p \in V_{\alpha}, p \notin V_{\beta}(\beta<\alpha), \alpha<\tau$, then we can choose $n$ such that $U_{n}(p) \subseteq V_{\alpha}$. Since $p \in\left(S_{n}^{1}(p)\right)^{\circ} \subseteq V_{n \alpha}$ for this $n$, we can choose $m$ satisfying $U_{m}(p) \subseteq V_{n \alpha}$. In consequence we have $S_{m}^{1}(p) \subseteq V_{m n \alpha}^{\prime}$. On the other hand, it follows from $p \notin V_{\beta}(\beta<\alpha)$ and from i) that $S_{n}^{2}(p) \frown$ $V_{n \beta}=\phi(\beta<\alpha)$. This implies

$$
S_{m}^{1}(p) \frown S_{n}^{2}(p) \subseteq V_{m n \alpha}^{\prime}-\underset{\beta<\alpha}{\smile} V_{n \beta}
$$

and consequently $p \in M_{m n \alpha}$, i.e. $\mathfrak{M}$ covers $R$. Since $\mathfrak{M}<\mathfrak{B}$ is obvious, we can conclude, from Michael's theorem, the paracompactness of $R$. Thus it follows from A. H. Stone's theorem ${ }^{5}$ that $R$ is fully normal.

To complete the proof, let us show that $\left\{S\left(p, \Im_{m}\right) \mid m=1,2, \cdots\right\}^{6)}$ for $\mathfrak{S}_{m}=\left\{\left(S_{m}^{2}(y)\right)^{\circ} \mid y \in R\right\}$ is a nbd basis of each point $p$ of $R$. Let $U(p)$ be an arbitrary nbd of $p$, and choose $n$ satisfying $U_{n}(x) \subseteq U(x)$, then we can find $m \geqq n$ with $U_{m}(x) \subseteq S_{n}^{1}(x)$. If $\left(S_{m}^{2}(y)\right)^{\circ} \ni x$, then considering $S_{m}^{2}(y) \frown S_{m}^{1}(x) \neq \phi$, we have $y \in U_{m}(x) \subseteq S_{n}^{1}(x)$ from i). Hence it follows from ii) that $S_{m}^{2}(y) \subseteq S_{n}^{2}(y) \subseteq U_{n}(x)$ because we can assume, without loss of generality, that $m \geqq n$ implies $S_{m}^{2}(y) \subseteq S_{n}^{2}(y)$. Therefore we have $S\left(x, \Im_{m}\right) \subseteq U(x)$, i.e. $\left\{S\left(p, \Im_{m}\right) \mid m=1,2, \cdots\right\}$ is a $\mathrm{nbd}$ basis of $p$. Thus we conclude the metrizability of $R$ by AlexandroffUrysohn's theorem.

We enumerate some of direct consequences of this theorem without proofs.

Theorem 2 (Yu. Smirnov [8] and the author [6]). A regular space $R$ is metrizable if and only if there exists an open basis $\mathfrak{B}=\underset{n=1}{\cup} \mathfrak{B}_{n}$ of $R$, where each $\mathfrak{V}_{n}$ is a locally finite collection of open sets.

Theorem 3 (K. Morita [5]). A $T_{1}$-space $R$ is metrizable if and only if there exists a countable collection $\left\{\mathfrak{\mho}_{n} \mid n=1,2, \cdots\right\}$ of locally finite closed coverings of $R$ such that $S\left(x, \mathfrak{\mho}_{n}\right) \subseteq U(x)$ for any nbd $U(x)$ of any point $x$ of $R$ and for some $n$.

Theorem 4 (R. H. Bing [1]). A regular space $R$ is metrizable if and only if there exists a countable collection $\left\{\mathfrak{U}_{n} \mid n=1,2, \cdots\right\}$ of open collections such that the sum of the closures of any subcollection of $\mathfrak{H}_{n}$ is closed and such that $\left\{S\left(p, \mathfrak{U}_{n}\right) \mid n=1,2, \cdots\right\}$ is a nbd basis of each point $p$ of $R$.

Theorem 5 (K. Morita [4]). A generalization of Alexandroff-

5) See [9].

6) $S\left(p, \mathfrak{S}_{m}\right)=\smile\left\{S \mid p \in S \in \mathbb{S}_{m}\right\}$. 
Urysohn's theorem. A $T_{1}$-space $R$ is metrizable if and only if there exists a countable collection $\left\{\mathfrak{U}_{n} \mid n=1,2, \cdots\right\}$ of open coverings such that $\left\{S\left(S\left(p, \mathfrak{U}_{n}\right), \mathfrak{U}_{m}\right) \mid m, n=1,2, \cdots\right\}$ is a nbd basis of each point $p$ of $R$.

Theorem 6 (A. H. Frink [2]). A $T_{1}$-space $R$ is metrizable if and only if one can assign a nbd basis $\left\{U_{n}(x) \mid n=1,2, \cdots\right\}$ for every point $x$ of $R$ such that for every $n$ and $p \in R$ there exists $m=m(n, x)$ satisfying the condition: $U_{m}(x) \frown U_{m}(y) \neq \phi$ implies $U_{m}(y) \subseteq U_{n}(x)$.

Theorem 7 (the author [7]). $A T_{1}$-space $R$ is metrizable if and only if there exists a family $\left\{f_{\alpha} \mid \alpha \in A\right\}$ of real valued continuous functions of $R$ such that

i) $\underset{\beta \in B}{\smile} f_{\beta}$ and $\underset{\beta \in B}{\underset{\beta}{(1)} f_{\beta}}$ are continuous for every $B \subseteq A$,

ii) for any nbd $U(x)$ of any point $x$ of $R$ there exist $\alpha \in A$ and $\varepsilon>0$ such that $f_{\alpha}(x)<\varepsilon, f_{\alpha}(y) \geqq \varepsilon(y \notin U(x))$.

Theorem 8. A T $T_{1}$-space $R$ is metrizable if and only if there exists a non-negative function $\varphi(x, y)$ of $R \times R$ satisfying

i) $\varphi(x, y)=\varphi(y, x)$

ii) $d(x, A)=\inf \{\varphi(x, y) \mid y \in A\}$ for every subset $A$ of $R$ is a continuous function of $x$,

iii) $\quad\left\{S_{n}(x) \mid n=1,2, \cdots\right\}$ for $S_{n}(x)=\{y \mid \varphi(x, y)<1 / n\}$ is a nbd basis of each point $x$ of $R .{ }^{7}$

\section{References}

[1] R. H. Bing: Metrization of topological spaces, Canadian Jour. Math., 3 (1951).

[2] A. H. Frink: Distance function and the metrization problem, Bull. Amer. Math. Soc., 43 (1937).

[3] E. Michael: A note on paracompact spaces, Proc. Amer. Math. Soc., 4, no. 3 (1953).

[4] K. Morita: On the simple extension of a space with respect to a uniformity. IV, Proc. Japan Acad., 27, no. 10 (1951).

[5] K. Morita: A condition for the metrizability of topological spaces and for $n$ dimensionality, Sic. Rep. Tokyo Kyoiku Daigaku, Sect. A, 5, no. 114 (1955).

[6] J. Nagata: On a necessary and sufficient condition of metrizability, Jour. Inst. Polytech. Osaka City Univ., Ser. A, 1, no. 2 (1950).

[7] J. Nagata: On coverings and continuous functions, Jour. Inst. Polytech. Osaka City Univ., Ser. A, 7, nos. 1-2 (1956).

[8] Yu. Smirnov: A necessary and sufficient condition for metrizability of topological space, Doklady Akad. Nauk SSSR. N.S., 77 (1951).

[9] A. H. Stone: Paracompactness and product spaces, Bull. Amer. Math. Soc., 54, no. 10 (1948).

7) This theorem is an extension of Collorary 6 of [7]. An extension of Lemma 2 in that paper is, also, deduced from Theorem 1. 